\title{
Myeloid leukemia-1 expression in benign and malignant melanocytic lesions
}

\author{
RONALD P.C. WONG ${ }^{1 *}$, SHAHRAM KHOSRAVI $^{1 *}$, MAGDALENA MARTINKA $^{2}$ and GANG LI ${ }^{1}$ \\ ${ }^{1}$ Department of Dermatology and Skin Science, Jack Bell Research Centre; ${ }^{2}$ Department of Pathology, Vancouver \\ Coastal Health Research Institute, University of British Columbia, Vancouver, British Columbia, Canada
}

Received November 9, 2007; Accepted December 18, 2007

\begin{abstract}
Myeloid leukemia-1 (Mcl-1) is an anti-apoptotic protein implicated in tumor progression. Its expression was found to be elevated in many types of human cancers and is correlated with tumor progression. The expression of Mcl-1 in melanoma is not fully understood. We investigated the expression of Mcl-1 in normal nevi, dysplastic nevi, primary melanoma and melanoma metastases by tissue microarray and immunohistochemistry. We found that Mcl-1 expression was significantly increased in dysplastic nevi, primary melanoma and melanoma metastases when compared to normal nevi, though the expression of Mcl-1 was decreased in metastatic melanoma when compared to dysplastic nevi. We did not find any correlation between Mcl-1 expression and melanoma patient survival. Our data suggest that Mcl-1 may play a critical role in the initiation of melanoma development.
\end{abstract}

\section{Introduction}

Melanoma is a life-threatening skin cancer, characterized by rapid metastasis and a resistance to conventional radio- and chemo-therapy. Although melanoma accounts for only $4 \%$ of all dermatological cancers, it is responsible for $80 \%$ of deaths from skin cancer (1). Melanomas at the early stages are curable with surgical excision (2). However, up to $20 \%$ of patients will develop metastatic tumors owing to its high capability of invasion and rapid metastasis to other organs (3). Only $14 \%$ of patients with metastatic melanoma survive for five years (1). Resistance to chemotherapy, with a response rate of $<20 \%$, is the major problem in treating melanoma $(4,5)$.

Apoptosis plays an important role in the process of tumor formation. The molecular components of apoptosis include

Correspondence to: Dr Gang Li, Jack Bell Research Centre, 2660 Oak Street, Vancouver, British Columbia V6H 3Z6, Canada

E-mail: gangli@interchange.ubc.ca

${ }^{*}$ Contributed equally

Key words: myeloid leukemia-1, melanoma, apoptosis, tissue microarray proapoptotic and antiapoptotic regulators (6). Members of the Bcl-2 family of proteins are key regulators of apoptosis (7). They include the proapoptotic $\mathrm{Bcl}$ homology domain 3 (BH3) only proteins (such as Bid, Bim, Bad, NOXA and PUMA) and antiapoptotic prosurvival proteins such as Bcl-2, Bcl-2like 1 (Bcl-XL) and Myeloid leukemia-1 (Mcl-1) proteins. $\mathrm{Bcl}-2$ associated $\mathrm{X}$ protein (BAX) and BAK are additional proapoptotic proteins that appear to be essential for the induction of apoptosis $(8,9)$. The antiapoptotic proteins bind the proapoptotic $\mathrm{BH} 3$ proteins in order to prevent the homodimerization of BAX and BAK and the subsequent depolarization of mitochondrial membrane potential $(10,11)$.

Mcl-1 was first found to be expressed in differential myeloid cells (12). Since then Mcl-1 has been shown to be expressed in epithelia such as prostate, breast, colon and lung (13). Mcl-1 was also detected in the adrenal cortical cells, sympathetic neurons, leydig cells of the testis, a subpopulation of cells in the pancreatic islets, granulosa lutein cells of the ovarian corpus luteum and cardiac and skeletal muscles (13).

The role of Mcl-1 in melanoma is not well understood. Zhuang et al showed increased Mcl-1 expression in thick primary melanoma and metastatic melanoma (14). Since these authors included only 10 cases of dysplastic nevi in their report, the role of Mcl-1 in the initial cell transformation of melanocytes is not clear. To elucidate the role of Mcl-1 in melanomagenesis, we employed tissue microarray (TMA) technology and studied Mcl-1 expression in 187 melanocytic lesions at different stages including 44 cases of dysplastic nevi by immunohistochemistry. For the first time, our study showed a higher expression of Mcl-1 in dysplastic nevi compared to normal nevi suggesting that the overexpression of Mcl-1 may be involved in melanoma initiation.

\section{Materials and methods}

Construction of TMA. The use of human skin tissues in this study was approved by the Medical Ethics Committee of the University of British Columbia and was performed according to the declaration of the Helsinki guidelines. Formalin-fixed, paraffin-embedded tissues from 13 human normal nevi, 44 dysplastic nevi, 83 primary melanomas and 47 metastatic melanomas were used in this study. All specimens are from the 1990 to 1998 archives of the Department of Pathology, Vancouver General Hospital. For each specimen, the most representative lesion area was selected and marked on the 

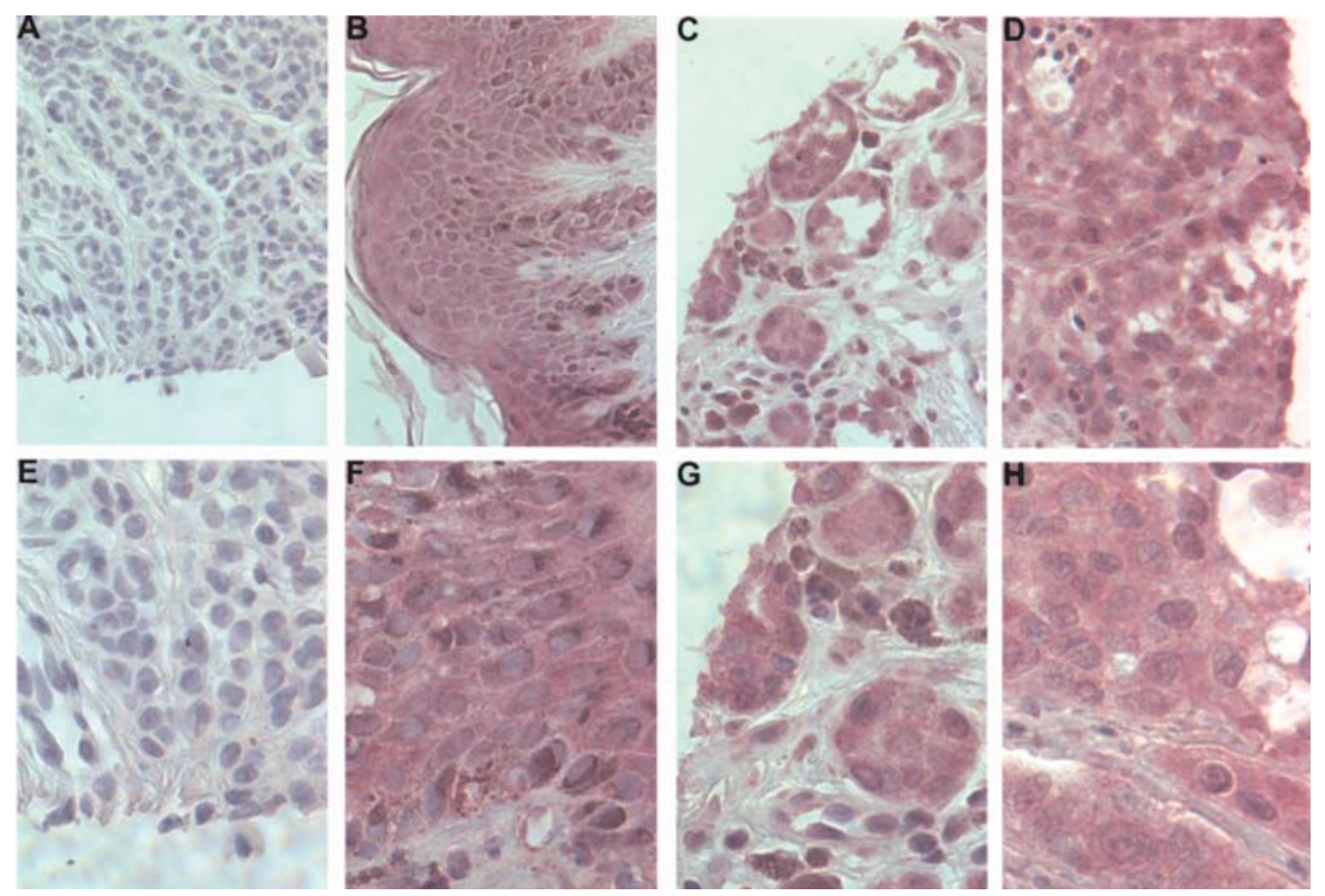

Figure 1. Representative images of Mcl-1 immunohistochemical staining in human melanocytic lesions. (A and E) Normal nevus with negative Mcl-1 expression. (B and F) Dysplastic nevus with strong Mcl-1 expression. (C and G) Primary melanoma with strong Mcl-1 expression. (D and H) Melanoma metastasis with strong Mcl-1 expression. Magnification x150 for A-D; x400 for E-H.

hematoxylin-eosin-stained slide. Duplicate 0.6-mm thick tissue cores were taken from each biopsy and the TMAs were assembled using a tissue-array instrument (Beecher Instruments, Silver Spring, MD). Using a Leica microtome (Leica Microsystems Inc, Bannockburn, IL), multiple 4- $\mu \mathrm{m}$ sections were cut and then transferred to adhesive coated slides using regular histology procedures. Hematoxylin and eosin were used to stain one section from each TMA. Other sections were kept at room temperature for immunohistochemical staining.

Immunohistochemistry of TMA. For deparaffinization, the TMA slides were heated at $55^{\circ} \mathrm{C}$ for $20 \mathrm{~min}$ followed by three 5 -min washes with xylene. The rehydration of tissues was performed by 5 -min washes in 100, 95 and $80 \%$ ethanol and distilled water. Antigen retrieval was performed by heating the samples at $95^{\circ} \mathrm{C}$ for $30 \mathrm{~min}$ in $10 \mathrm{mM}$ sodium citrate (pH 6.0). Endogenous peroxidase activity was blocked by incubation in $3 \%$ hydrogen peroxide for $30 \mathrm{~min}$. After $30 \mathrm{~min}$ of blocking with the universal blocking serum (Dako Diagnostics, Missisauga, ON, Canada) the sections were incubated with a primary antibody (1:100 dilution; Santa Cruz, CA, USA) at $4^{\circ} \mathrm{C}$ overnight. Then the sections were incubated for $30 \mathrm{~min}$ each with a biotin-labeled secondary antibody and then streptavidin-peroxidase (Dako Diagnostics). The samples were then developed using 3,3'-diaminobenzidine substrate (Vector Laboratories, Burlington, ON, Canada) and counterstained with hematoxylin. Dehydration was then performed following a standard procedure and the slides were sealed with coverslips. Negative controls were performed by omitting the Mcl-1 antibody during the primary antibody incubation.
Evaluation of immunostaining. The Mcl-1 staining was examined by one dermatologist and two other observers simultaneously and a consensus score was reached for each core. Based on the intensity of the staining, the positive reaction of Mcl-1 was scored into four grades of $0,1,2$ and 3 . The percentage of Mcl-1-positive cells was scored into five groups of $0(0 \%), 1$ (1-25\%), 2 (26-50\%), $3(51-75 \%)$ and $4(76-100 \%)$. In the event of a discrepancy between duplicated cores, the higher score from the two cores was used as the final score. The multiplication of the intensity and percentage scores is used as the final staining score. The final score for the staining was defined as negative (0), weak (1-4), moderate (5-8) or strong (9-12).

Statistical analysis. The quantitative differences of Mcl-1 staining at different stages of melanoma were compared using the $\chi^{2}$ test. The $\chi^{2}$ test was also used to evaluate the correlation between Mcl-1 expression and the clinicopathological parameters of primary melanoma patients such as age, gender, tumor thickness, ulceration, tumor location, histological subtype and American Joint Committee on Cancer (AJCC) stage. The correlations between Mcl-1 staining and patient survival were evaluated using the Kaplan-Meier method and log-rank test. A P-value of $<0.05$ was considered significant.

\section{Results}

Clinicopathological features of TMAs. The TMA employed in the study consists of 13 cases of normal nevi, 44 cases of dysplastic nevi, 83 cases of primary melanoma and 47 cases of melanoma metastases. For the 83 cases of primary melanoma, 
Table I. Mcl-1 staining and clinicopathological characteristics of 87 primary melanomas.

\begin{tabular}{|c|c|c|c|c|c|c|}
\hline & \multicolumn{4}{|c|}{ Mcl-1 staining score } & \multirow[b]{2}{*}{ Total } & \multirow[b]{2}{*}{ P-value } \\
\hline & 0 & 1 & 2 & 3 & & \\
\hline \multicolumn{7}{|l|}{ Age } \\
\hline$\leq 57$ & $2(5 \%)$ & $13(32 \%)$ & $19(46 \%)$ & $7(17 \%)$ & 41 & $\mathrm{P}>0.05$ \\
\hline$>57$ & $5(12 \%)$ & $14(33 \%)$ & $17(40 \%)$ & $6(14 \%)$ & 42 & $\mathrm{P}>0.05$ \\
\hline \multicolumn{7}{|l|}{ Gender } \\
\hline Male & $4(8 \%)$ & $14(27 \%)$ & $24(47 \%)$ & $9(18 \%)$ & 51 & $\mathrm{P}>0.05$ \\
\hline Female & $3(9 \%)$ & $13(41 \%)$ & $12(38 \%)$ & $4(13 \%)$ & 32 & $P>0.05$ \\
\hline \multicolumn{7}{|c|}{ Tumor thickness (mm) } \\
\hline$\leq 1$ & $2(8 \%)$ & $7(29 \%)$ & $11(46 \%)$ & $4(17 \%)$ & 24 & $\mathrm{P}>0.05$ \\
\hline $1.01-2$ & $2(7 \%)$ & $10(34 \%)$ & $12(41 \%)$ & $5(17 \%)$ & 29 & $\mathrm{P}>0.05$ \\
\hline $2.01-4$ & $2(15 \%)$ & $5(38 \%)$ & $4(31 \%)$ & $2(15 \%)$ & 13 & $\mathrm{P}>0.05$ \\
\hline$>4$ & $1(6 \%)$ & $5(29 \%)$ & $9(53 \%)$ & $2(12 \%)$ & 17 & $\mathrm{P}>0.05$ \\
\hline \multicolumn{7}{|l|}{ Ulceration } \\
\hline Absent & $7(11 \%)$ & $20(30 \%)$ & $29(44 \%)$ & $10(15 \%)$ & 66 & $\mathrm{P}>0.05$ \\
\hline Present & $0(0 \%)$ & $7(41 \%)$ & $7(41 \%)$ & $3(18 \%)$ & 17 & $P>0.05$ \\
\hline \multicolumn{7}{|l|}{ Tumor subtype } \\
\hline SSM & $2(6 \%)$ & $11(31 \%)$ & $17(47 \%)$ & $6(17 \%)$ & 36 & $\mathrm{P}>0.05$ \\
\hline LMM & $2(13 \%)$ & $5(33 \%)$ & $7(47 \%)$ & $1(7 \%)$ & 15 & $P>0.05$ \\
\hline Other & $3(9 \%)$ & $11(34 \%)$ & $12(38 \%)$ & $6(19 \%)$ & 32 & $P>0.05$ \\
\hline \multicolumn{7}{|l|}{ Site } \\
\hline Sun-protected & $6(9 \%)$ & $19(28 \%)$ & $30(45 \%)$ & $12(18 \%)$ & 67 & $P>0.05$ \\
\hline Sun-exposed & $1(6 \%)$ & $8(50 \%)$ & $6(38 \%)$ & $1(6 \%)$ & 16 & $\mathrm{P}>0.05$ \\
\hline
\end{tabular}

${ }^{\mathrm{a}} \chi^{2}$ test. ${ }^{\mathrm{b}}$ Sun-protected sites: trunk, arm, leg and feet. Sun-exposed sites: head and neck.

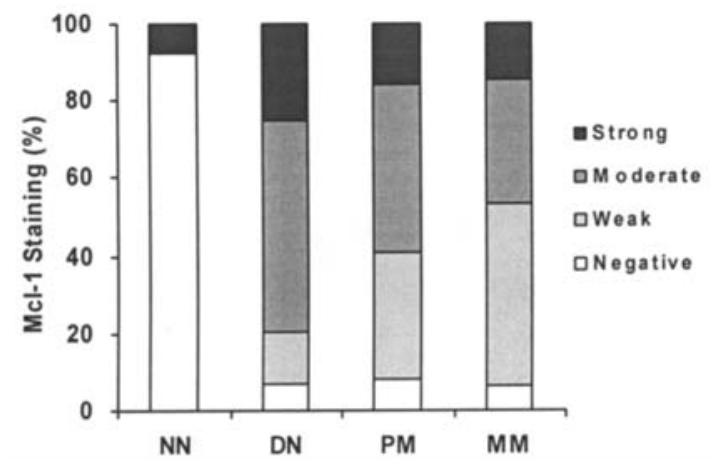

Figure 2. Mcl-1 expression in normal nevi (NN), dysplastic nevi (DN), primary melanoma (PM) and melanoma metastases (MM). Negative staining (0); weak staining (1-4); moderate staining (5-8); and strong staining (9-12). A significantly higher expression in Mcl-1 was found in DN, PM and MM when compared to $\mathrm{NN}\left(\mathrm{P}<0.001, \chi^{2}\right.$ test $)$ and decreased Mcl-1 expression in $\mathrm{MM}$ and when compared to $\mathrm{DN}\left(\mathrm{P}<0.005, \chi^{2}\right.$ test $)$.

32 were male and 51 were female, with ages ranging from 21 to 93 years $($ median $=58)$. For primary melanoma staging, we used Breslow thickness as our criteria (15) for evaluating Mcl-1 expression: 24 tumors were $\leq 1.0 \mathrm{~mm}, 29$ were 1.01 $2.0 \mathrm{~mm}, 13$ were $2.01-4.0 \mathrm{~mm}$ and 17 were $>4.0 \mathrm{~mm}$. Sixtyseven tumors were located in sun-exposed (head and neck) sites, while 16 tumors were located in sun-protected sites (other). Ulceration was observed in 17 cases (Table I).
Increased Mcl-1 expression in dysplastic nevi and melanomas. We examined the overall staining in normal nevi, dysplastic nevi, primary melanoma and metastatic melanoma by immunohistochemistry (Fig. 1). Mcl-1 showed moderate to strong cytoplasmic staining in 8, 80, 59 and $47 \%$ of normal nevi, dysplastic nevi, primary melanoma and metastatic melanoma, respectively. A significant difference in Mcl-1 staining was observed between normal nevi and dysplastic nevi, primary melanoma or metastatic melanoma $(\mathrm{P}<0.001$, $\chi^{2}$ test). A significant difference in Mcl-1 staining was also observed between dysplastic nevi and metastatic melanoma $\left(\mathrm{P}<0.005, \chi^{2}\right.$ test $)$. However, the difference of Mcl-1 staining between dysplastic nevi and primary melanoma $(\mathrm{P}=0.105$, $\chi^{2}$ test) and between primary and metastatic melanomas were not significant ( $\mathrm{P}=0.423, \chi^{2}$ test) (Fig. 2).

Correlation of Mcl-1 expression with clinicopathological parameters and melanoma patient survival. AJCC stages, tumor thickness and ulceration are known to be important prognostic markers for patients with primary melanoma. We studied if Mcl-1 expression correlates with these markers. We did not find a significant correlation between Mcl-1 expression and AJCC stages, tumor thickness or ulceration (Table I), neither any association between Mcl-1 expression and other clinicopathological parameters such as age, gender, and tumor subtypes (Table I). We then examined if Mcl-1 expression was associated with the survival of patients with 

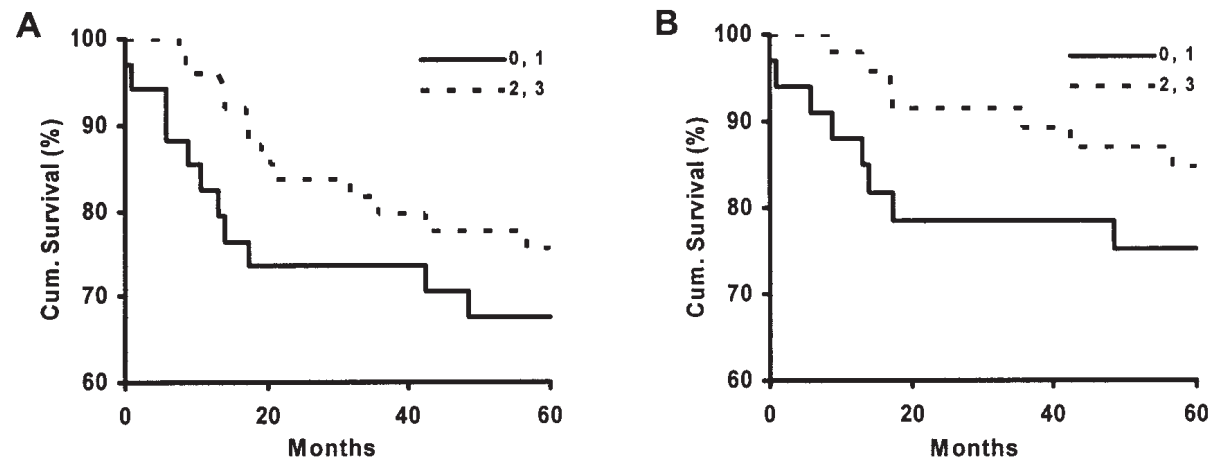

Figure 3. Correlation between Mcl-1 expression and 5-year survival of primary melanoma patients. There is no significant correlation of Mcl-1 expression with (A) overall 5-year survival ( $\mathrm{P}>0.05$, log-rank test) and (B) disease-specific 5-year survival ( $\mathrm{P}>0.05, \log$-rank test).

primary melanoma by Kaplan-Meier survival analysis. We divided Mcl-1 staining into two groups, negative to weak and moderate to strong. Our results showed that Mcl-1 expression did not correlate with 5-year overall (Fig. 3A) and diseasespecific (Fig. 3B) patient survival in primary melanoma $(\mathrm{P}>0.05, \log$-rank test).

\section{Discussion}

Impairment of apoptosis is a critical step in tumorigenesis $(16,17)$. p53 plays a central role in the regulation of apoptosis and protects cells from tumorigenesis $(18,19)$. However, alterations in the TP53 gene only occur in 1-5\% and $11-25 \%$ of primary and metastatic melanomas, respectively (20), indicating that the alteration of other components in the apoptotic pathway may be important for tumorigenesis . Apoptosis is tightly controlled by the ratio of pro-survival (e.g. Bcl-2, Bcl-XL, Bcl-w, Mcl-1 and A1) and apoptotic (e.g. BAX, BAK, Bim, Bad, NOXA and PUMA) Bcl-2 family members (21). Mcl-1 is a pro-survival factor which functions through the formation of heterodimer with BAX and/or BAK to inhibit mitochondria-mediated apoptosis (22-24). Mcl-1 expression is associated with a higher grade of tumors (14,25-28). The study of Mcl-1 expression in melanoma is limited. We sought to investigate the expression of Mcl-1 at different stages of melanocytic lesions using tissue microarray technology and immnohistochemistry. We found that Mcl-1 expression increased significantly in dysplastic nevi, primary melanoma and metastatic melanoma when compared to normal nevi. Notably, we also found that the expression of Mcl-1 significantly decreased in metastatic melanoma when compared to dysplastic nevi (Fig. 2). This implies the critical role of Mcl-1 in the initiation of melanomagenesis. This is in accordance with a previous study, which also showed an elevated Mcl-1 expression in melanoma when compared to the normal nevi biopsies (29). However, we did not find any significant correlation of Mcl-1 expression with other clinicopathological parameters (Table I) and patient survival (Fig. 3). According to a recent study by Zhuang et al, Mcl-1 expression increased with the progression of melanoma and strong Mcl-1 expression correlated with worse patient survival (14). Although, it in part agrees with our finding, we noted a major discrepancy in that we found a significant increase of Mcl-1 expression in dysplastic nevi when compared to normal nevi which is not the case in the report by Zhuang et al. Zhuang et al tested only 10 cases of dysplastic nevi compared to 44 cases in our study, which may account for the different findings. Different antibodies used in the immunohistochemical studies could also be the cause of the discrepancy.

Apoptosis plays a critical role in tumorigenesis. In fact, it has been shown that the activation of an oncogene alone could not lead to malignant transformation. Secondary oncogenic lesions that suppress apoptosis and/or enhance proliferation are needed for transformation to malignancy (30-32). We found that Mcl-1 is strongly expressed specifically in dysplastic nevi. This suggests the potential importance of Mcl-1 in the initiation of melanoma formation. We postulate that an increased expression of Mcl-1 acts as a secondary oncogenic signal to protect transformed cells from apoptosis induced by oncogenic transformation. It has been demonstrated in vitro that Mcl-1 protects cells from c-Myc-induced apoptosis (23). The activation of Myc alone is insufficient to drive tumorigenesis, though the inactivation of BAX eliminates all restraints to the oncogenic potential of Myc and allows the progression of tumors in vivo (33). Myc is found to be overexpressed in many cancers including melanoma $(34,35)$. Our data corroborate with the idea that Mcl-1 plays a critical role in the maintenance of transformed melanocytic lesions.

Notably, we also found that Mcl-1 expression decreased in metastatic melanomas when compared to dysplastic nevi. This could be explained by the heterogeneous nature of tumors. Alterations of other components in the apoptotic machinery may compensate for Mcl-1 expression during melanoma progression. Our group has demonstrated that the expression of other apoptotic proteins such as PUMA (36), XAF-1 (37), Apaf-1 (38) and Bim (39) were altered in melanoma. This may also be the reason for the fact that no significant correlation was found between Mcl-1 expression and patient survival because the expression of other apoptotic proteins may also be involved.

Many factors could explain the up-regulation of Mcl-1 in melanoma. Stat3 is an important transcriptional activator of Mcl-1 and has been reported to up-regulate Mcl-1 (40). Other transcription factors such as microphthalmia transcription factor (MITF) and the activating protein $2 \alpha$ (AP- $2 \alpha$ ) have also been suggested to have a certain role in the regulation of Mcl-1 expression (14). The expression of Mcl-1 could also be altered at the post-translational level. Mcl-1 was found to be degraded through proteasome in a GSK3ß-dependent manner and Akt 
is known to phosphorylate and inactivate GSK3ß $(41,42)$. We previously showed that $p$-Akt is elevated in melanoma (43). Therefore, Mcl-1 may be elevated through the inhibition of degradation by an activated Akt in melanoma.

Melanoma progression is a complicated multi-stage process with alterations of many molecular events. We observed that the elevation of Mcl-1 expression was very common in dysplastic nevi highlighting the importance of Mcl-1 specifically in melanoma initiation. With previous studies on the expression of other apoptotic proteins in melanoma, this study sheds light on the role of apoptosis in melanoma initiation and the potential of targeting Mcl-1 in the prevention of melanoma.

\section{Acknowledgements}

We thank Dr M. Germain for providing reagents and D. Ngan and D. Chen for their technical assistance. This work was supported by the Canadian Dermatology Foundation.

\section{References}

1. Miller AJ and Mihm MC Jr: Melanoma. N Engl J Med 355: 51-65, 2006.

2. Balch CM, Buzaid AC, Soong SJ, et al: Final version of the American Joint Committee on Cancer staging system for cutaneous melanoma. J Clin Oncol 19: 3635-3648, 2001.

3. Houghton AN and Polsky D: Focus on melanoma. Cancer Cell 2: 275-278, 2002.

4. Gilchrest BA, Eller MS, Geller AC and Yaar M: The pathogenesis of melanoma induced by ultraviolet radiation. N Engl J Med 340: 1341-1348, 1999

5. Li Y and McClay EF: Systemic chemotherapy for the treatment of metastatic melanoma. Semin Oncol 29: 413-426, 2002.

6. Hussein MR, Haemel AK and Wood GS: Apoptosis and melanoma: molecular mechanisms. J Pathol 199: 275-288, 2003.

7. Cory S and Adams JM: The Bcl2 family: regulators of the cellular life-or-death switch. Nat Rev Cancer 2: 647-656, 2002.

8. Cheng EH, Wei MC, Weiler S, et al: BCL-2, BCL-X(L) sequester $\mathrm{BH} 3$ domain-only molecules preventing $\mathrm{BAX}$ - and BAK-mediated mitochondrial apoptosis. Mol Cell 8: 705-711, 2001.

9. Wei MC, Zong WX, Cheng EH, et al: Proapoptotic BAX and BAK: a requisite gateway to mitochondrial dysfunction and death. Science 292: 727-730, 2001.

10. Willis SN, Chen L, Dewson G, et al: Proapoptotic Bak is sequestered by Mcl-1 and Bcl-xL, but not Bcl-2, until displaced by BH3-only proteins. Genes Dev 19: 1294-1305, 2005.

11. Chen L, Willis SN, Wei A, et al: Differential targeting of prosurvival $\mathrm{Bcl}-2$ proteins by their $\mathrm{BH} 3$-only ligands allows complementary apoptotic function. Mol Cell 17: 393-403, 2005 .

12. Kozopas KM, Yang T, Buchan HL, Zhou P and Craig RW: MCL1, a gene expressed in programmed myeloid cell differentiation, has sequence similarity to BCL2. Proc Natl Acad Sci USA 90: 3516-3520, 1993.

13. Krajewski S, Bodrug S, Krajewska M, et al: Immunohistochemical analysis of Mcl-1 protein in human tissues. Differential regulation of $\mathrm{Mcl}-1$ and $\mathrm{Bcl}-2$ protein production suggests a unique role for Mcl-1 in control of programmed cell death in vivo. Am J Pathol 146: 1309-1319, 1995.

14. Zhuang L, Lee CS, Scolyer RA, et al: Mcl-1, Bcl-XL and Stat3 expression are associated with progression of melanoma whereas Bcl-2, AP-2 and MITF levels decrease during progression of melanoma. Mod Pathol 20: 416-426, 2007.

15. Balch CM, Soong SJ, Gershenwald JE, et al: Prognostic factors analysis of 17,600 melanoma patients: validation of the American Joint Committee on Cancer melanoma staging system. J Clin Oncol 19: 3622-3634, 2001.

16. Vaux DL, Cory S and Adams JM: Bcl-2 gene promotes haemopoietic cell survival and cooperates with c-myc to immortalize pre-B cells. Nature 335: 440-442, 1988.

17. Strasser A, Harris AW, Bath ML and Cory S: Novel primitive lymphoid tumours induced in transgenic mice by cooperation between myc and bcl-2. Nature 348: 331-333, 1990.
18. Oren M: Decision making by $\mathrm{p} 53$ : life, death and cancer. Cell Death Differ 10: 431-442, 2003.

19. Fridman JS and Lowe SW: Control of apoptosis by p53. Oncogene 22: 9030-9040, 2003.

20. Akslen LA, Monstad SE, Larsen B, Straume O and Ogreid D: Frequent mutations of the p53 gene in cutaneous melanoma of the nodular type. Int J Cancer 79: 91-95, 1998.

21. Adams JM and Cory S: The Bcl-2 apoptotic switch in cancer development and therapy. Oncogene 26: 1324-1337, 2007.

22. Cuconati A, Mukherjee C, Perez D and White E: DNA damage response and MCL-1 destruction initiate apoptosis in adenovirusinfected cells. Genes Dev 17: 2922-2932, 2003.

23. Reynolds JE, Yang T, Qian L, et al: Mcl-1, a member of the Bcl-2 family, delays apoptosis induced by c-Myc overexpression in Chinese hamster ovary cells. Cancer Res 54: 6348-6352, 1994.

24. Tao W, Kurschner C and Morgan JI: Modulation of cell death in yeast by the Bcl-2 family of proteins. J Biol Chem 272: 15547-15552, 1997

25. Khoury JD, Medeiros LJ, Rassidakis GZ, McDonnell TJ, Abruzzo LV and Lai R: Expression of Mcl-1 in mantle cell lymphoma is associated with high-grade morphology, a high proliferative state, and p53 overexpression. J Pathol 199: 90-97, 2003.

26. Michels J, Foria V, Mead B, et al: Immunohistochemical analysis of the antiapoptotic Mcl-1 and Bcl-2 proteins in follicular lymphoma. Br J Haematol 132: 743-746, 2006.

27. Cho-Vega JH, Rassidakis GZ, Admirand JH, et al: MCL-1 expression in B-cell non-Hodgkin's lymphomas. Hum Pathol 35: 1095-1100, 2004.

28. Shigemasa K, Katoh O, Shiroyama Y, et al: Increased MCL-1 expression is associated with poor prognosis in ovarian carcinomas. Jpn J Cancer Res 93: 542-550, 2002.

29. Tang L, Tron VA, Reed JC, et al: Expression of apoptosis regulators in cutaneous malignant melanoma. Clin Cancer Res 4: 1865-1871, 1998.

30. Letai A, Sorcinelli MD, Beard C and Korsmeyer SJ: Antiapoptotic BCL-2 is required for maintenance of a model leukemia. Cancer Cell 6: 241-249, 2004.

31. Pelengaris S, Khan M and Evan GI: Suppression of Myc-induced apoptosis in beta cells exposes multiple oncogenic properties of Myc and triggers carcinogenic progression. Cell 109: 321-334, 2002.

32. Schmitt CA, McCurrach ME, de Stanchina E, Wallace-Brodeur RR and Lowe SW: INK4a/ARF mutations accelerate lymphomagenesis and promote chemoresistance by disabling p53. Genes Dev 13: 2670-2677, 1999.

33. Dansen TB, Whitfield J, Rostker F, Brown-Swigart L and Evan GI: Specific requirement for Bax, not Bak, in Myc-induced apoptosis and tumor suppression in vivo. J Biol Chem 281: 10890-10895, 2006.

34. Nesbit CE, Tersak JM and Prochownik EV: MYC oncogenes and human neoplastic disease. Oncogene 18: 3004-3016, 1999.

35. Ramsden AJ, Grover R, Chana J, Tulley P, Sanders R and Wilson GD: A prospective analysis of c-myc oncoprotein levels as a prognostic marker in malignant melanoma. J Plast Reconstr Aesthet Surg 60: 626-630, 2007.

36. Karst AM, Dai DL, Martinka M and Li G: PUMA expression is significantly reduced in human cutaneous melanomas. Oncogene 24: 1111-1116, 2005

37. $\mathrm{Ng} \mathrm{KC}$, Campos EI, Martinka M and Li G: XAF1 expression is significantly reduced in human melanoma. J Invest Dermatol 123: 1127-1134, 2004

38. Dai DL, Martinka M, Bush JA and Li G: Reduced Apaf-1 expression in human cutaneous melanomas. Br J Cancer 91: 1089-1095, 2004.

39. Dai DL, Wang Y, Liu M, Martinka M and Li G: Bim expression is reduced in human cutaneous melanomas. J Invest Dermatol 128: 403-407, 2008

40. Jing $\mathrm{N}$ and Tweardy DJ: Targeting Stat 3 in cancer therapy. Anticancer Drugs 16: 601-607, 2005.

41. Letai A: Growth factor withdrawal and apoptosis: the middle game. Mol Cell 21: 728-730, 2006.

42. Ding Q, He X, Xia W, et al: Myeloid cell leukemia-1 inversely correlates with glycogen synthase kinase-3beta activity and associates with poor prognosis in human breast cancer. Cancer Res 67: 4564-4571, 2007

43. Dai DL, Martinka M and Li G: Prognostic significance of activated Akt expression in melanoma: a clinicopathologic study of 292 cases. J Clin Oncol 23: 1473-1482, 2005. 